(c) American Dairy Science Association, 2004.

\title{
Technical Note: Comparison of Chromatographic Profile of Glycomacropeptide from Cheese Whey Isolated Using Different Methods
}

Esther W. Y. Li and Yoshinori Mine

Department of Food Science, University of Guelph, Guelph, Ontario N1G 2W1, Canada

\section{ABSTRACT}

Glycomacropeptide (GMP) has heterogeneous carbohydrates, and this attributes to its various biological activities. In this study, we compared the chromatographic profiles of GMP isolated by three methods (trichloracetic acid fractionation, ethanol precipitation, and ultrafiltration) from whey protein isolate (WPI). Seven sharp heterogeneous GMP peaks were eluted from GMP prepared by ethanol precipitation and ultrafiltration using Mono $\mathrm{Q}$ anionic chromatography, while only 5 peaks were seen in TCA treated sample. The TCA pretreatment recovered only sialoGMP (glycosylated) and eliminated all contaminated proteins; however, the recovery rate was the lowest (6.7\% of the initial WPI). Ethanol precipitation recovered $20.4 \%$ of GMP from WPI and $75.7 \%$ was glycosylated, but the heating process might lead to degradation of glycosidic residues. Ultrafiltration was found to be the most effective in recovering GMP. The recovery rate was $33.9 \%$ with $81.6 \%$ sialo-GMP. We concluded that carbohydrate profile of GMP varied widely and depended on the isolation method. Based on the high recovery of sialo-GMP, the combination of ultrafiltration and anionic chromatography might be a suitable and practical approach on an industrial scale.

(Key words: glycomacropeptide, whey protein isolate, carbohydrate, HPLC)

Abbreviation key: GMP = glycomacropeptide, UF = ultrafiltration, $\mathbf{W P I}=$ whey protein isolate.

Glycomacropeptide (GMP) present in cheese whey is a C-terminal glycopeptide released from $\kappa$-CN by the action of chymosin at ${ }^{105} \mathrm{Phe}-{ }^{106} \mathrm{Met}$ during cheese making (Eigel et al., 1984). It is the sole milk glycopeptide and contains various amounts of covalently attached oligosaccharides, including $\mathrm{N}$-acetylneur-

Received October 26, 2002.

Accepted February 27, 2003.

Corresponding author: Y. Mine; e-mail: ymine@uoguelph.ca. aminic acids (sialic acids), galactose, and $N$-acetylgalactosamine (Saito et al, 1991). Many researchers have discovered that GMP exhibits various biological activities, such as suppression of gastric secretion, promotion of bifido-bacterial growth, binding of cholera and Escherichia coli enterotoxin, inhibition of viral and bacterial adhesion, and modulation of immune system (Brody, 2000). The multifunctional bioactivities of GMP might be attributed to the carbohydrate chains, but the degree to which the carbohydrate moiety remains attached to GMP and its role in the biological functions of GMP has not been completely revealed (Dziuba and Minkiewicz, 1996). Because of its unique carbohydrate composition and biological activities, GMP is thought to be a potential ingredient for functional foods and nutraceuticals, and thus much attention has been given to the development of techniques to isolate and purify GMP from cheese whey. Glycomacropeptide has been isolated and purified from cheese whey or rennet casein by using various methods, such as TCA pretreatment (Morr and Seo, 1988), ethanol precipitation (Saito et al., 1991), and ultrafiltration (Kawasaki et al., 1993; Chu et al., 1996). Although many of GMP isolation methods have been described, few commercially viable technologies have been reported (Xu et al., 2000), and the effect of different isolation methods on GMP carbohydrate profiles has not yet been evaluated. Our study was thus undertaken to compare the above-three isolation methods of GMP from whey protein isolate (WPI) on the effect of its carbohydrate profile and to provide an effective isolating method that is suitable for industrial needs.

\section{MATERIALS AND METHODS}

Isolation of GMP using a TCA pretreatment was followed by the procedures described by Morr and Seo (1988). Twenty milliliters of 5\% (wt/vol) WPI (Protein Fractionation Inc, Toronto, Canada) was dissolved in $12 \%$ (vol/vol) TCA. Precipitate was removed by centrifuge at $5000 \times g$ for $20 \mathrm{~min}$. Supernatant that contained GMP was then dialyzed against deionized water for 2 $\mathrm{d}$ with $3.5-\mathrm{kDa}$ cut-off membrane (Fisher Scientific, 
Nepean, Ontario, Canada). In ethanol precipitation method, $20 \mathrm{~mL}$ of $5 \%$ WPI (pH 3.5) was heat at $98^{\circ} \mathrm{C}$ for $1 \mathrm{~h}$, followed by rapid cooling to $4^{\circ} \mathrm{C}$, and precipitated with $50 \%$ (vol/vol) cold ethanol. The solution was then readjusted to $\mathrm{pH} 9.0$ by $1.0 \mathrm{~N} \mathrm{NaOH}$, and flocculent material was removed by centrifuge at $3000 \times g$ for $30 \mathrm{~min}$, leaving a supernatant containing GMP (Saito et al., 1991). Ultrafiltration was also documented to isolate GMP with a modification of the method described before (Kawasaki et al, 1993). Twenty milliliters of 5\% WPI at pH 3.5 was filtered through Whatman No. 1 filter (Fisher Scientific), and then subjected to Amicon 8050 ultrafiltrater (Millipore Corporation, Bedford, MA) fitted with 50-kDa MW cutoff ultrafiltration membrane (Millipore Corporation). After the first ultrafiltration, permeate was readjusted to $\mathrm{pH} 7.0$ with $1.0 \mathrm{~N} \mathrm{NaOH}$. The second and third ultrafiltration was carried out by the same ultrafiltration (UF) membrane to retain GMP in the retentate.

All crude GMP were then subjected to Mono Q HR5/ 5 anion-exchange chromatography (Pharmacia Biotech., Uppsala, Sweden) with a linear gradient of $\mathrm{NaCl}$ from 0 to $1.0 M$, using HPLC (Waters, Ventura, CA) to analyze the heterogeneity of each GMP component. The column was equilibrated and eluted with $20 \mathrm{mM}$ Tris-HCl buffer at $\mathrm{pH} 8.0$ at flow rate of $1.0 \mathrm{~mL} / \mathrm{min}$. The effluent was monitored at $214 \mathrm{~nm}$.

\section{RESULTS AND DISCUSSION}

Glycomacropeptide could be recovered from WPI by all three methods described above. The apparent molecular weight was found to be 20 to $24 \mathrm{kDa}$ from $15 \%$ SDS polyacrylamide electrophoresis (data not shown). Although it was far above the theoretical MW of $7 \mathrm{kDa}$, the apparent molecular weight of GMP was comparable to those reported previously. According to a previous study of GMP by gel chromatography, molecular weight of GMP aggregate was $\mathrm{pH}$ dependent. Molecular weight was distributed from 20 to $50 \mathrm{kDa}$ at $\mathrm{pH} 7$ and from 10 to $30 \mathrm{kDa}$ at $\mathrm{pH} 3.5$ (Kawasaki et al., 1993). The heterogeneous sugar chains that attached to the GMP molecule also contributed to the apparent molecular weight of GMP. Several studies have reported that at least 5 heterogeneous sugar chains were identified from bovine GMP (Flat et al., 1972; Saito et al, 1992). The biological activities of GMP are thought to depend on the content and structure of these sugar moieties (Dziuba et al., 1991).

Figure 1 illustrated the HPLC profile of GMP prepared by TCA pretreatment, ethanol precipitation, and UF. Seven sharp GMP peaks were eluted from GMP prepared by ethanol precipitation and UF, whereas only 6 peaks were identified in the TCA-

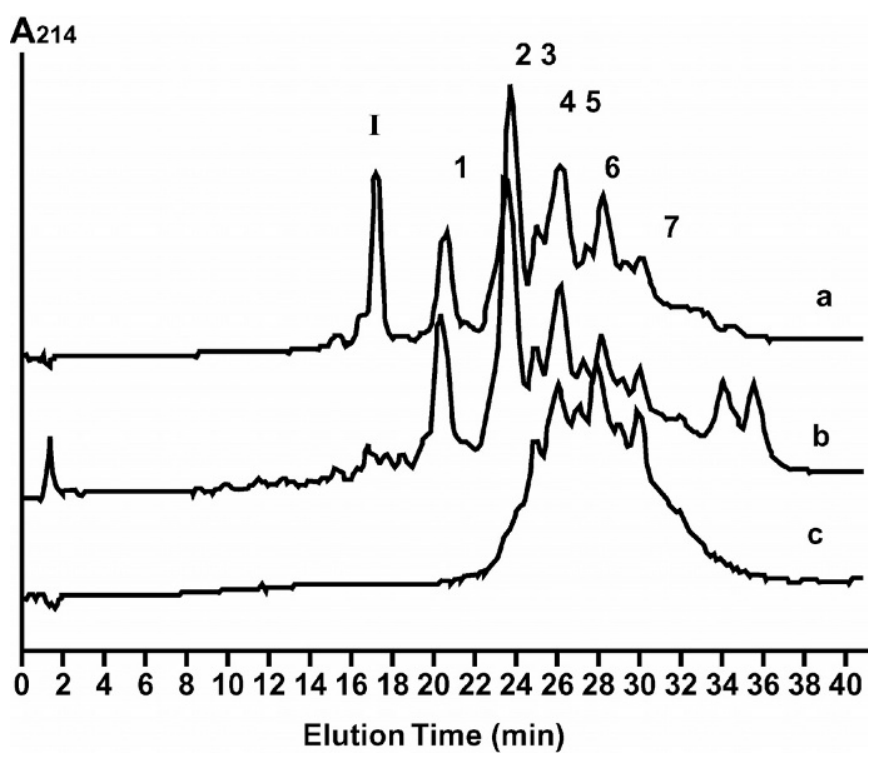

Figure 1. Elution profile of glycomacropeptide isolated from whey protein isolate by (a) ultrafiltration, (b) ethanol precipitation, and (c) TCA pretreatment under Mono Q HR5/5 anionic HPLC in $20 \mathrm{mM}$ Tris-HCl buffer at $\mathrm{pH}$ 8.0. I; impurity.

treated sample. The multiple elution peaks could be due to the heterogeneous sugar chains. This result was consistent with previous reports (Saito et al., 1991). The first peak was previously identified as an asialoGMP (nonglycosylated), and followed by 6 heterogeneous sialo-GMP (glycosylated) peaks (Kawakami et al., 1992). Table 1 summarized the percentage of each peak to the total recovered GMP isolated by different methods with different carbohydrate compositions in each GMP peak fraction. Because these heterogeneous sugar chains were thought to be responsible for various biological activities of GMP, high recovery of sialoGMP would be of great interest for nutraceutical research.

The TCA pretreatment successfully isolated carbohydrate-rich GMP fractions. All of the recovered GMP was glycosylated. Although no other contaminant peaks were observed in TCA pretreated GMP, only 5 blurred peaks were eluted. However, the recovery rate was the lowest among the three, only $6.7 \%$ of the initial WPI. The recovery rate of GMP was calculated relative to the initial protein concentration (GMP concentration/initial WPI concentration) (Kawasaki et al., 1993). The TCA treatment eliminated asialo-GMP and the first sialo-GMP fraction, probably because TCA could precipitate small sugar chains due to their low hydropilicity. It was reported that the different asialoand sialo-GMP have different sensitivities to TCA precipitation (Leonil and Molle, 1991). In addition, the presence of the large TCA peaks precluded the visual- 
Table 1. Percentage of glycomacropeptide (GMP) fractions by different isolation methods from whey protein isolate.

\begin{tabular}{|c|c|c|c|c|c|c|c|c|}
\hline Peak & 1 & 2 & 3 & 4 & 5 & 6 & 7 & $\begin{array}{l}\text { Total } \\
\text { glycosylated } \\
\text { GMP }\end{array}$ \\
\hline \multicolumn{9}{|c|}{ Method: (\% of each peak/total GMP) ${ }^{1}$} \\
\hline $\mathrm{EtOH}$ & 24.3 & 46.2 & 3.1 & 14.1 & 1.3 & 7.7 & 3.3 & 75.7 \\
\hline TCA & - & - & 12.1 & 23.1 & 5.0 & 36.2 & 23.5 & 100 \\
\hline \multicolumn{9}{|c|}{ Carbohydrate and phosphorus content $(\mathrm{mol} / \mathrm{mol})^{2}$} \\
\hline GaINAc & 0 & 1 & 1 & 2 & 2 & 2 & 3 & \\
\hline Phosphorus & 0 & 1 & 1 & 1 & 1 & 1 & 1 & \\
\hline
\end{tabular}

${ }^{1}$ The data are means of three repetitions. UF; ultrafiltration, EtOH; ethanol precipitation, TCA; trichloroacetic acid fractionation. NeuAc = $N$-acetylneuraminic acid (sialic acid); Gal $=$ galactose and GalNAc $=N$-acetylgalactosamine.

${ }^{2}$ Data were adapted by Kawakami et al., 1992.

ization of possible peptides that might be eluted in the same retention time. Thus, the pretreatment of whey with TCA might lead to poor estimation of GMP content unless the differential solubility of GMP in TCA was taken into consideration (Kawakami et al., 1992).

Ethanol precipitation recovered both asialo- and sialo-GMP simultaneously. Glycomacropeptide (20.4\%) was recovered, and $75.7 \%$ was glycosylated. Although this method had a reasonably high recovery and purity of sialo-GMP, heat coagulation of whey protein during the isolation process might lead to partial hydrolysis of $\kappa$-CN with liberation of GMP and degrade glycosidic residues (van Hooydonk et al., 1987). Heat coagulation degraded some of the sugar chains in sialo-GMP and gave rise to a higher ratio of asialo-GMP (Table 1).

Among the 3 isolation methods, UF was found to have the highest recovery of GMP (33.9\%) with $81.6 \%$ sialo-GMP. Ultrafiltration provided the most convenient and effective way to eliminate the pretreatment of whey with TCA and ethanol, while providing genuine content of both asialo- and sialo-GMP simultaneously. In fact, membrane filtration is the most widely used method presently on industrial basis for recovery of whey proteins such as $\alpha$-lactalbumin and $\beta$-LG (Rossano et al., 2001).

In conclusion, different isolation and preparation methods of GMP resulted in different chromatographic profile in GMP. These heterogeneous carbohydrate chains were the unique characteristics of GMP, not found in the remaining part ( 1 to 105) of $\kappa-\mathrm{CN}$. Each of the GMP fractions with different carbohydrate chains might be responsible for different biological functions. The result obtained from this study provides useful information for further investigation as to structurefunction studies of biological activities of GMP. In addition, UF method was found to be the most effective in GMP separation compared with TCA and ethanol pretreatment method. Not only of its high recovery and high purity of sialo-GMP, it also eliminated the pretreatment of sample with TCA and ethanol while retaining the structure of the carbohydrate structures. The combination of UF and an anionic chromatography may be suitable and practical for industrial production of GMP.

\section{ACKNOWLEDGMENTS}

This work was supported by grants from the Natural Sciences and Engineering Research Council of Canada (NSERC), Agriculture and Agri-Food Canada, and Dairy Farmers of Ontario.

\section{REFERENCES}

Brody, E. P. 2000. Biological activities of bovine glycomacropeptide. Br. J. Nutr. 84(Suppl. 1):S39-S46.

Chu, L., A. Macleod, and L. Ozimek. 1996. Isolation of glycomacropeptide from sodium caseinate hydrolysate solution by ultrafiltration. Milchwissenschaft 51:303-306.

Dziuba, J., and P. Minkiewicz. 1996. Influence of glycosylation on micelle-stabilizing ability and biological properties of the C-terminal fragments of cow's $\kappa$-casein. Int. Dairy J. 6:1017-1044.

Dziuba, J., P. Minkiewicz, and S. Buczel. 1991. The influence of $N$ acetylneuraminic acid (NANA) content in casein on milk functional properties. Appl. Biol. Commun. 1:372-378.

Eigel, M. N., J. E. Buttler, C. A. Ernstorm, H. M. Farrell, V. R. Harwalker, R. Jenness, and R. M. Whitney. 1984. Nomenclature of proteins of cow's milk: Fifth revision. J. Dairy Sci. 67:1599-1631.

Flat, A. M., C. Alais, and P. Jolles. 1972. The amino acid and carbohydrate sequences of the short glycopeptide isolated from bovine $\kappa$-casein. Eur. J. Biochem. 27:408-412.

Kawakami, H., Y. Kawasaki, S. Dosako, M. Tanimoto, and I. Nakajima. 1992. Determination of $\kappa$-casein glycomacropeptide by high performance liquid chromatography without trichloroacetic acid pretreatment. Milchwissenscaft 47:688-693.

Kawasaki, Y., H. Kawakami, M. Tanimoto, S. Dosako, A. Tomizawa, M. Kotake, and I. Nakajima. 1993. pH-Dependent molecular weight changes of $\kappa$-casein glycomacropeptide and its preparation by ultrafiltration. Milchwissenschaft 48:191-195.

Leonil, J., and D. Molle. 1991. A method for determination of macropeptide by cation-exchange fast protein liquid chromatography and its use for following action of chymosin in milk. J. Dairy Res. 58:321-328. 
Morr, C. V., and A. Seo. 1988. Fractionation and characterization of glycomacropeptide from caseinate and skim milk hydrolysates. J. Food Sci. 53:80-87.

Rossano R., A. D'Elia, and P. Riccio. 2001. One step separation from lactose: Recovery and purification of major cheese whey protein by hydroxyapatite: A flexible procedure suitable for small- and medium-scale preparations. Protein Expr. Purif. 21:165-169.

Saito, T., A. Yamaji, and T. Itoh. 1991. New isolation method of GMP from sweet whey. J. Dairy Sci. 74:2831-2837.
Saito, T., and T. Itoh. 1992. Variations and distribution of O-glycosidically linked sugar chains in bovine $\kappa$-casein. J. Dairy Sci. 75:1768-1774.

van Hooydonk, A. C. M., and P. Walstra. 1987. Interpretation of kinetics of renneting reaction in milk. Neth. Milk Dairy J. 41:19-47.

Xu, Y., R. Sleigh, J. Hourigan, and R. Johnson. 2000. Separation of bovine immunoglobulin $\mathrm{G}$ and glycomacropeptide from dairy whey. Process Biochem. 36:393-399. 